\section{Neutropenia asociada al uso de cefepime en pa- cientes pediátricos con fibrosis quística}

\author{
Rubén Hernández', Luis Delpiano', Jorge Amador', \\ Mariana Arias $^{2}$ y Juan Carrasco Delgado'
}

Neutropenia associated with the use of cefepime in pediatric patients with cystic fibrosis

Pulmonary exacerbations of infectious cause are one of the major complications in patients with cystic fibrosis (CF). These are associated with a progressive increase in morbidity and mortality. The treatment depending on the isolated microorganism. The $\beta$-lactam antibiotics are generally used which are not exempt from adverse reactions. Next, two report of neutropenia cases are described after prolonged use of cefepime in CF patients.

Keywords: $\beta$-lactams; cefepime; neutropenia; cystic fibrosis.

Palabras clave: $\beta$-lactámicos; cefepime; neutropenia; fibrosis quística.

\section{Introducción}

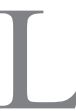

os pacientes con fibrosis quística (FQ) utilizan con frecuencia antimicrobianos $\beta$-lactámicos debido a cuadros de exacerbaciones respiratorias infecciosas causadas principalmente por Staphylococcus aureus y/o Pseudomonas aeruginosa. Los $\beta$-lactámicos más utilizados son cloxacilina, ceftazidima y cefepime. En esta patología los tratamientos antimicrobianos son, en general, prolongados, lo que favorece la aparición de reacciones adversas como las alteraciones hematológicas y, en particular, la neutropenia ${ }^{1-3}$.

La familia de los $\beta$-lactámicos incluye a las penicilinas, cefalosporinas, monobactam y carbapenémicos, los cuales tienen en común presentar en su estructura química un anillo $\beta$-lactámico. Su mecanismo de acción está dado por la inhibición de la síntesis de la pared celular bacteriana secundaria a su unión con la enzima transpeptidasa llamada proteína fijadora de penicilina (PBP) $;{ }^{4,5}$. Dada su acción bactericida y elevado perfil de seguridad, esta familia de antimicrobianos es la más utilizada en pediatría ${ }^{6,7}$.

La neutropenia asociada a los antimicrobianos $\beta$-lactámicos fue descrita por primera vez en 1946 asociada al uso de penicilina. Desde entonces, y dado el aumento de la farmacovigilancia, existen varias publicaciones sobre esta asociación ${ }^{8-10}$. Se presentan dos casos clínicos pediátricos de neutropenia asociada al uso de $\beta$-lactámicos. La aparición de esta entidad estaría relacionada con factores como: el uso de altas dosis, tratamientos

'Hospital Clínico San Borja Arriarán. Santiago, Chile.

${ }^{2}$ Clínica Las Condes. Santiago, Chile.

Sin conflictos de interés. Sin financiamiento.

Recibido: 6 de julio de 2018/ Aceptado: 4 de enero de 2019

Correspondencia a:

Rubén Hernández $\mathrm{M}$.

rthernan@uc.cl prolongados (más de 10 días), la edad (mayor aparición en niños) y disfunción hepática y renal ${ }^{11-13}$.

\section{Caso clínico 1}

Paciente de sexo femenino de 2 años de edad con diagnóstico de fibrosis quística en la etapa neonatal. Tenía antecedentes de insuficiencia pancreática, mal incremento ponderal y exacerbaciones respiratorias con cultivos de secreción bronquial con $S$. aureus sensible a meticilina (SAMS). Se internó por una exacerbación respiratoria por lo que inició terapia antimicrobiana triasociada con cloxacilina $(50 \mathrm{mg} / \mathrm{kg} / \mathrm{dosis}$ cada $6 \mathrm{~h})$, ceftazidima $(50 \mathrm{mg} /$ $\mathrm{kg} /$ dosis cada $8 \mathrm{~h}$ ) y amikacina (30 mg/ $\mathrm{kg}$ una vez al día). Al ingreso la función renal era normal, PCR de $3,8 \mathrm{mg} / \mathrm{dl}$ y leucocitos de 9.310 céls $/ \mathrm{mm}^{3}$ (RAN: 5.030 neutrófilos $/ \mathrm{mm}^{3}$ ). Por falta de disponibilidad de ceftazidima en nuestro hospital, se cambió a cefepime $50 \mathrm{mg} / \mathrm{kg} / \mathrm{dosis}$ cada $8 \mathrm{~h}$. El cultivo de secreción bronquial fue positivo para $S$. aureus y $P$. aeruginosa multisensibles, manteniéndose la terapia triasociada.

La paciente evolucionó de forma satisfactoria. Tras 16 días de tratamiento se controló un hemograma, pesquisándose un recuento de leucocitos 1.800 céls $/ \mathrm{mm}^{3}$ y RAN 43 céls $/ \mathrm{mm}^{3}$. Se sospechó una reacción adversa asociada a $\beta$-lactámicos, por lo que se suspendió cloxacilina y cefepime, cambiando a levofloxacina i.v. $20 \mathrm{mg} / \mathrm{kg} /$ día. A los dos días, el hemograma de control mostró un aumento del recuento leucocitario a 3.500 céls $/ \mathrm{mm}^{3}$, manteniendo la neutropenia con RAN de $165 \mathrm{cel} / \mathrm{mm}^{3}$, lo que se recuperó al sexto día de suspensión de cefepime (Gráfico 1).

\section{Caso clínico 2}

Paciente de sexo masculino, 4 años de edad, con diagnóstico de fibrosis quística a los dos años de edad tras varios episodios de neumonías. Tenía antecedentes de colonización crónica por SAMS, daño pulmonar crónico con bronquiectasias, insuficiencia pancreática, neurofibromatosis tipo 1 y oxígeno dependencia.

Se internó por fiebre y aumento de las secreciones respiratorias. Se obtuvo cultivo de secreción bronquial y se inició tratamiento antibacteriano con ceftazidima $(50 \mathrm{mg} / \mathrm{kg} / \mathrm{dosis}$ cada $8 \mathrm{~h})$, amikacina $(20 \mathrm{mg} / \mathrm{kg}$ una vez al día) y cotrimoxazol (6 mg/kg/dosis cada $8 \mathrm{~h}$, en base a trimetoprim), dado un último cultivo de secreción bronquial con Stenotrophomonas maltophilia. El cultivo de secreción bronquial tomado al ingreso resultó positivo a $P$. aeruginosa multisensible. Por desabastecimiento de ceftazidima, se cambió esquema a cefepime $(50 \mathrm{mg} / \mathrm{kg} / \mathrm{dosis}$ cada $8 \mathrm{~h})$ más amikacina, suspendiéndose cotrimoxazol. La amikacina se suspendió luego de completar 14 días de terapia.

Al día 16 de cefepime, el paciente presentó fiebre de $39^{\circ} \mathrm{C}$. Se tomaron cultivos y exámenes de laboratorio: PCR de $7 \mathrm{mg} / \mathrm{dl}$, leucocitos 12.700/ $\mathrm{mm}^{3}$ (segmentados 69\%), función renal y hepática normal. Se realizó una ecocardiografía que mostró el catéter venoso central en aurícula derecha con engrosamiento de 9 × $5 \mathrm{~mm}$ en la punta del dispositivo, sugerente de una endocarditis infecciosa. Se agregó al esquema antimicrobiano vancomicina $(15 \mathrm{mg} / \mathrm{kg} / \mathrm{dosis}$ cada $6 \mathrm{~h}$ ) y anfotericina B complejo lipídico $(3 \mathrm{mg} / \mathrm{kg} /$ día) y se retiró el catéter.

Al día 20 de cefepime el paciente volvió a presentar fiebre. Todos los cultivos resultaron negativos. Se controló un hemograma destacando una leucopenia de 2.530 céls $/ \mathrm{mm}^{3}$ (segmentados $28 \%$ ) y un RAN de 700 céls/ $\mathrm{mm}^{3}$. La PCR fue $24,3 \mathrm{mg} / \mathrm{dl}$. Se controló una ecocardiografía que resultó normal. Habiéndose descartado una endocarditis se suspendió vancomicina 
y anfotericina B, así como cefepime por sospecha de ser causante de la neutropenia. Tres días luego de la suspensión de cefepime se normalizó el recuento de leucocitos (8.960 céls $/ \mathrm{mm}^{3}$ ) y el RAN (6.270 céls $/ \mathrm{mm}^{3}$ ) (Gráfico 2).

Se realizó el análisis de causalidad de ambas reacciones adversas utilizando el algoritmo de Naranjo. En ambas reacciones adversas se obtuvieron 7 puntos, clasificándolas como probable.

\section{Discusión}

No existen estudios de incidencia de neutropenia asociada al uso de $\beta$-lactámicos. La mayoría de las publicaciones corresponden a descripciones de casos. Además, en muchas ocasiones el punto de corte para definir neutropenia varía entre los diferentes autores, agregándose el hecho que algunos estudios están enfocados solo en un grupo particular de $\beta$-lactámicos, principalmente las penicilinas ${ }^{12}$.

En niños con FQ existe una serie retrospectiva de 38 pacientes (mediana de edad 14 años) que recibieron piperacilina-tazobactam, con una duración media de tratamiento de 12,5 días. En el análisis se encontró solo un evento de neutropenia asociado a trombocitopenia, que se recuperó tras cuatro días de suspensión del antimicrobiano. En nuestra comunicación, también se observó que los dos pacientes desarrollaron una disminución en los leucocitos y plaquetas ${ }^{14}$.

Taniuchi y cols. reportaron una neutropenia autoinmune asociado al uso de $\beta$-lactámicos (flomoxef y cefotiam) en una paciente pediátrico. Fue de aparición temprana (cinco días de iniciado el tratamiento) con una duración de dos meses. El mecanismo de producción de la neutropenia fue de carácter inmunológico, ya que los autores evidenciaron un aumento en la producción de anticuerpos contra los granulocitos, los cuales fueron disminuyendo tras la suspensión de los antibacterianos $^{15}$.

Existen dos mecanismos propuestos para explicar la neutropenia por $\beta$-lactámicos. El primero consiste en una toxicidad medular directa, efecto descrito por Neftel y cols. En esta investigación se expusieron distintos $\beta$-lactámicos a cultivos de medula ósea, resultando una inhibición dosis dependiente en la formación de colonias mieloides. Además, se evidenció que las cefalosporinas generaron una inhibición hasta 25 veces más potente que las penicilinas semisintéticas. Este estudio también concluyó que la inhibición se revertía tras retirar el antimicrobiano del cultivo ${ }^{16}$. Otro estudio desarrollado por Du y cols. demostró que los $\beta$-lactámicos son capaces de inhibir la ADN polimerasa en cultivos celulares de células mieloides ${ }^{17}$.

El segundo mecanismo sería inmunológico, cuya hipótesis es avalada por la aparición de anticuerpos anti-penicilina tras la administración de $\beta$-lactámicos. Estos anticuerpos interaccionan con los granulocitos y plaquetas generando la neutropenia y trombocitopenia. También, se ha descrito que la administración de $\beta$-lactámicos podría inducir la aparición de anticuerpos antigranulocitos, generando una disminución de estos ${ }^{15,18}$.

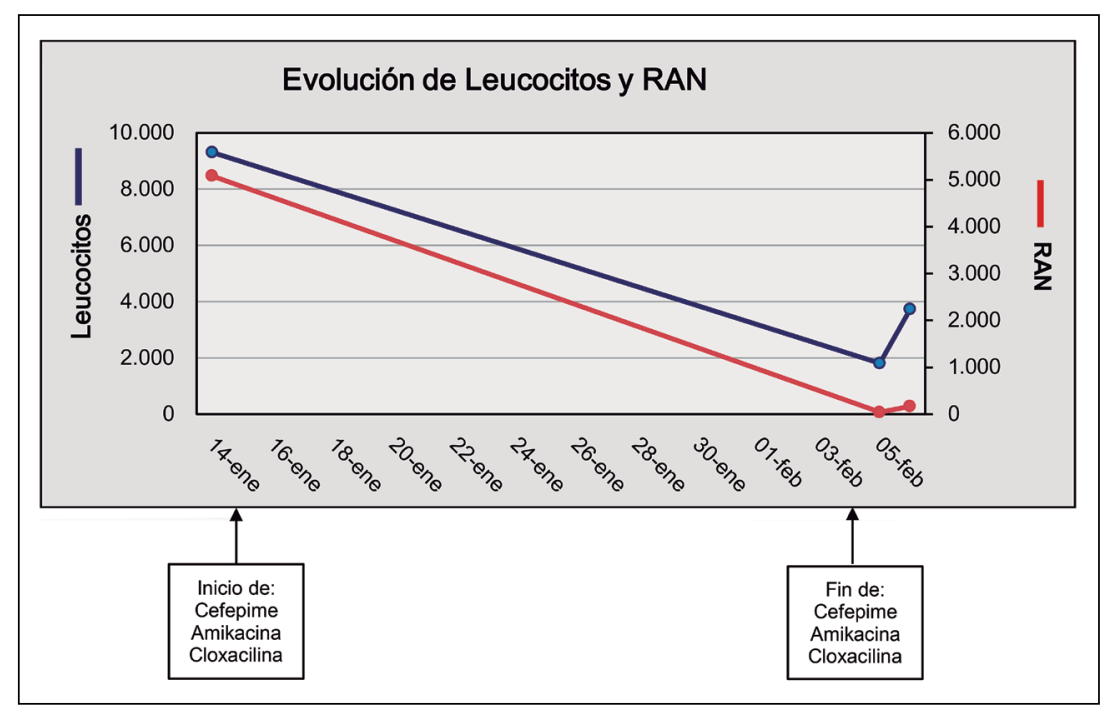

Gráfico 1. Evolución del recuento de leucocitos en hemograma del caso 1.

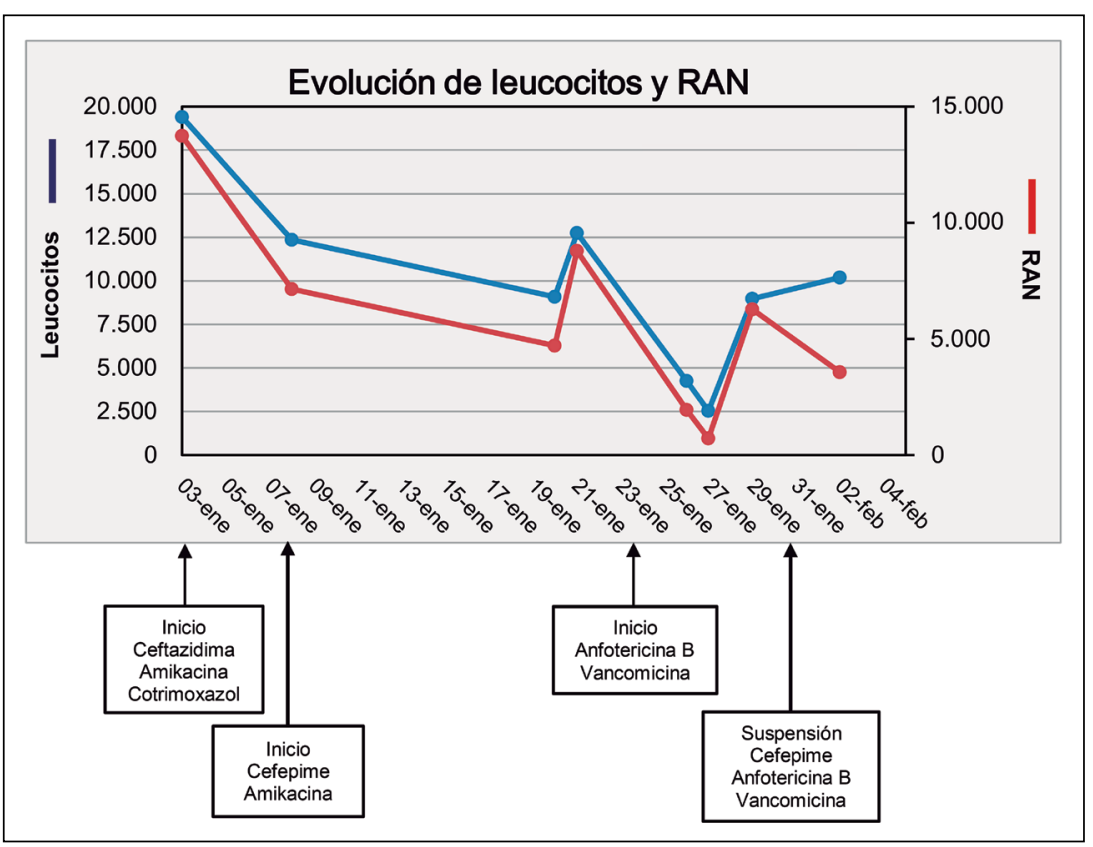

Gráfico 2. Evolución del recuento de leucocitos en hemograma del paciente caso 2.

Entre los factores de riesgo para la aparición de la neutropenia se encuentra la dosis acumulativa diaria de $\beta$-lactámicos. En los estudios de Olaison, en 50\% de los pacientes con endocarditis que recibían 27.000.000 UI diarias de penicilina sódica, desarrollaban neutropenia vs solo $14 \%$ en aquellos que recibían 21.000 .000 UI diarios de penicilina sódica $^{19-20}$. El segundo factor es el tiempo de tratamiento del antimicrobiano, ya que en la mayoría de los casos publicados de pacientes adultos la neutropenia aparece a los 14 días de tratamiento ${ }^{12,15,21}$. En nuestros casos descritos se asoció a la terapia prolongada, día 19 y 24 de terapia; respectivamente. Ambos pacientes recibieron $150 \mathrm{mg} / \mathrm{kg} / \mathrm{día}$ de cefepime, que es la dosis recomendada por distintas fuentes de información ${ }^{22}$. 
El pronóstico de esta reacción adversa es bueno, ya que la mayoría de los casos se resuelve en forma rápida, no más allá de cinco días tras la suspensión del antimicrobiano. En nuestro primer paciente hubo recuperación tras tres días del retiro de cefepime. Por otra parte, se han descrito buenos resultados con el uso de factores estimulantes de colonias de granulocitos; sin embargo, no es una recomendación de rutina ${ }^{12,21,24}$.

En relación a la reexposición a un antimicrobiano $\beta$-lactámico en un paciente que ya presentó neutropenia, se ha descrito que entre $15-30 \%$ de ellos vuelve a desarrollar neutropenia. En estos casos, los factores de riesgo son los mencionados anteriormente ${ }^{19,26}$.

Cabe destacar que en el caso 2 se descartó una infección viral como causa de la neutropenia. En el caso 1 se desestimó una infección debido a su buen estado general y la temporalidad de la reacción adversa y el uso de cefepime. En el paciente 2, al realizar el análisis con el algoritmo de Naranjo se determinó que la anfotericina B es un medicamento concomitante en la generación de la neutropenia, pero su frecuencia es menor en comparación a los $\beta$-lactámicos ${ }^{27}$.

Finalmente, la farmacovigilancia alcanza una mayor relevancia en estos casos debido a que los pacientes pediátricos no son parte habitual de los estudios desarrollados antes de aprobación de los medicamentos. Además, esta reacción adversa es frecuentemente de aparición tardía, problema mayor debido a que muchos de los estudios premercado de los antimicrobianos no incluyen una duración mayor a los 14 días $^{24}$.

Comunicando estos dos casos de neutropenia asociada al uso prolongado de cefepime, reforzamos el alerta a los médicos clínicos, para que exista vigilancia en relación a la aparición de este efecto adverso cuando se indique a pacientes por periodos prolongados.

\section{Resumen}

Las exacerbaciones pulmonares de causa infecciosa son una de las mayores complicaciones en los pacientes con fibrosis quística (FQ). Estas se asocian a un progresivo aumento en la morbilidad y mortalidad. El tratamiento antimicrobiano se realiza dependiendo del microorganismo aislado. Con frecuencia se utilizan antimicrobianos $\beta$-lactámicos, los cuales no están exentos de reacciones adversas. A continuación, se describen dos casos de neutropenia tras el uso prolongado de cefepime en pacientes con FQ.

\section{Referencias bibliográficas}

1.- Flume P A, Mogayzel P J, Robinson K A, Goss C H, Rosenblatt R L, Kuhn R J, et al. Cystic fibrosis pulmonary guidelines: treatment of pulmonary exacerbations. Am J Respir Crit Care Med 2009; 180: 802-08. DOI: $10.1164 / \mathrm{rccm} .200812-1845 \mathrm{PP}$.

2.- Zobell J T, Young D C, Waters C D, Ampofo K, Cash J, Marshall B C, et al. A survey of the utilization of antipseudomonal betalactam therapy in cystic fibrosis patients. Pediatr Pulmonol 2011; 46: 987-90. DOI: 10.1002/ ppul.21467.

3.- Murphy M F, Metcalfe P, Grint P C, Green A R, Knowles S, Amess J A, et al. Cephalosporin-induced immune neutropenia. Br J Haematol 1985; 59: 9-14.

4.- Aminov R. History of antimicrobial drug discovery: Major classes and health impact. Biochem. Pharmacol 2017; 133: 4-19. DOI: 10.1016/j. bcp.2016.10.001.
5.- Calvo J, Martínez-Martínez L. Antimicrobial mechanisms of action. Enferm Infecc Microbiol Clin 2009; 27:44-52. DOI: 10.1016/j.eimc.2008.11.001.

6.- Chavez-Bueno S, Stull T L. Antibacterial agents in pediatrics. Infect Dis Clin North Am 2009; 23: 865-80, viii. DOI: 10.1016/j.idc.2009.06.011.

7.- Rodrigo C. Uso de los antimicrobianos en la población pediátrica. Enferm Infecc Microbiol Clin 2010; 28: 310-20. DOI: 10.1016/j.eimc.2010.03.001.

8.- Spain D M, Clark T B. A case of agranulocytosis occurring during the course of penicillin therapy. Ann Intern Med 1946; 25: 732.

9.- Vial T, Bailly H, Perault-Pochat M C, Default A, Boulay C, Chouchana L, et al. Beta-lactam-induced severe neutropaenia: a descriptive study. Fundam Clin Pharmacol 2018. DOI: 10.1111/fcp.12419 [Epub ahead of print].

10.- Medrano-Casique N, Tong H, Borobia A, Carcas A, Frías J, Ramírez Elena. Nonchemotherapy drug-induced agranulocytosis in children detected by a prospective pharmacovigilance program. Pediatr Hematol Oncol 2016; 33: 441-56. DOI: 10.1080/08880018.2016.1234523.

11.- Lang R, Lishner M, Ravid M. Adverse reactions to prolonged treatment with high doses of carbenicillin and ureidopenicillins. Rev Infect Dis 1991; 13: 68-72.

12.- Peralta G, Sánchez-Santiago M B. Neutropenia secundaria a betalactámicos. Una vieja compañera olvidada. Enferm Infecc Microbiol Clin 2005; 23: 485-91.

13.- Singh N, Yu V L, Mieles L A, Wagener M M. $\beta$-Lactam antibiotic-induced leukopenia in severe hepatic dysfunction: risk factors and implications for dosing in patients with liver disease. Am J Med 1993; 94: 251-6.

14.- Reichardt P, Handrick W, Linke A, Schille R, Kiess W. Leukocytopenia, thrombocytopenia and fever related to piperacillin/tazobactam treatment-a retrospective analysis in 38 children with cystic fibrosis. Infection 1999; 27: 355-6.

15.- Taniuchi S, Masuda M, Yamamoto A, Hasui M, Tsuji S, Takahashi H, et al. Two cases of autoimmune neutropenia possibly induced by beta-lactam antibiotics in infants. J Pediatr Hematol Oncol 2000; 22: 533-8.

16.- Neftel K, Hauser S, Müller M. Inhibition of granulopoiesis in vivo and in vitro by beta-lactam antibiotics. J Infect Dis 1985; 152: 90-8.

17.- Do U H, Neftel A, Spadari S, Hubscher U. Betalactam antibiotics interfere with eukaryotic DNA-replication by inhibiting DNA polymerase alpha. Nucleic Acids Res 1987; 15: 10495-506.

18.- Murphy M F, Riordan T, Minchinton R M, Chapman J F, Amess J A, Shaw E J, et al. Demonstration of an immune-mediated mechanism of penicillininduced neutropenia and thrornbocytopenia. Br J Haematol 1983; 55: 15560.

19.- Olaison L, Alestig K. A prospective study of neutropenia induced by high doses of $\beta$-lactam antibiotics. J Antimicrob Chemother 1990; 25: 449-53.

20.- Olaison L, Belin L, Hogevik H, Alestig K. Incidence of beta-lactam-induced delayed hypersensitivity and neutropenia during treatment of infective endocarditis. Arch Intern Med 1999; 159: 607-15.

21.- Wong B B, Ko G J. Neutropenia in patients receiving long-term cefepime therapy for osteomyelitis. Am J Health Syst Pharm 2003; 60: 2229-32.

22.- www. micromedex.com. Fecha de acceso: 4 de julio de 2018.

23.- Beauchesne M, Shalansky S. Nonchemotherapy drug-induced agranulocytosis: a review of 118 patients treated with colony-stimulating factors. Pharmacotherapy 1999; 19: 299-305.

24.- Peralta F G, Sánchez M B, Roiz M P, Pena M A, Tejero M A, Arjona R. Incidence of neutropenia during treatment of bone-related infections with piperacillin-tazobactam. Clin Infect Dis 2003; 37: 1568-72.

25.- Andres E, Maloisel F. Antibiotic-induced agranulocitosis: a monocentric study of 21 cases. Arch Intern Med 2001; 161: 2619.

26.- Schmid L, Heit W, Flury R. Agranulocytosis associated with semisynthetic penicillins and cephalosporins. Report of 7 cases. Blut 1984; 48: 11-8.

27.- Pick A M, Nystrom K K. Nonchemotherapy drug-induced neutropenia and agranulocytosis: could medications be the culprit? J Pharm Pract 2014; 27 : 447-52. DOI: 10.1177/0897190014546115. 